\title{
Training Needs of Faculty of Physical Education, Al- Azhar University staff members in Light of 21st Century Skills
}

\section{Dr. Alsayed Abdulaziz Alsayed Eisa ${ }^{(*)}$ \\ Introduction}

Twenty first century have numerous cognitive economic and technological transformations, which highly affected human beings in terms of their capabilities, efficiencies and skills. It is noticeable that countries possess knowledge, economic and technological components can get excellence in different areas of life, and those do not possess these ingredients remain in need of other to be subordinate to it always. Due to these transformations, importance to develop capabilities of university staff members emerged, where staff member is of the most important inputs that university depend on in achieving its goals. He entrusted with human energy teaching and conduct scientific researches that contribute to knowledge advancement and development, in addition to find solutions to problems facing society in $\mathrm{w}$ hich he lives. (Mishra, 2007, p.5)

Therefore, Universities should give concern to staff members, provide all that support their performance, achieve development for all their roles in university, and effectively contribute in accessing various global changes (Badr, 2015, p.84)

There is no doubt that the process of identifying training needs for university staff member represents the first and the most important step in university educational process success and achieving university aims, which is an objective starting point for training programs planning and design for staff members.

In this context, Sahu (2010) argues that training needs is the term required to move from existing to desired performance level, this need appear where there is a gap between what individual can

\footnotetext{
${ }^{(*)}$ Asst. professor, Department of curricula and teaching methods, Faculty of physical education, Al-Azhar University
}

Assiut Journal For Sport Science Arts 
do and what able to do. (p. 175)

should be

Identifying training needs is important because it help in planning training programs, study problems and shortcomings in individuals performance, and examine the gap between organization aims and current situation. It is also guides training to the correct and appropriate direction; it clarifies deficiencies, problems and difficulties encountered in the process of individuals' performance of their work tasks. (Al-Buhairi, 2011, p.135)

Many

literature and educational studies such as (Morrison \& Lowther, 2002) and (Chuang, 2002) study emphasized the importance of identifying university staff members training needs and train them to meet the informational and technical acceleration witnessed in contemporary reality. Additional consequences and responsibilities faced by staff member, which called for devel opment of their preparation programs in light of $21^{\text {st }}$ century skills that work on linking theoretical information with practical application in functional way based on innovation and employing technological techniques in the educational process, $21^{\text {st }}$ century teacher is the factor that determine education quality at all levels.

The twenty first century skills is of trends that began to gain attention by educational skills, in order to support students in the university and career life in terms of mastery of both content and skills. Concern of these skills in all disciplines started by Partnership for $21 \mathrm{st}$ Century Skills, which established through partnership between Department of Education in United States and group of businesses such as Microsoft Corporation, National Association for Education, Microsoft and National Education Association. This partnership now is of important world leaders of and the skills of the $21^{\text {st }} \quad$ century skills development and teaching.

Twenty-first skills are the skills necessary to ensure that learners are ready to learn, innovate, life, work and the proper use of information, media and 
technology in the $21^{\text {st }}$ century. (Shalabi, 2014, p.6)

Twenty-first century

skills enable learners to life and work in knowledge society era, where effective communication with others depends on technology, increasing need for possessing untypical problem-solving skills and find innovative solutions to these problems. This called to reconsider teacher skills needed to be prepared appropriately to perform the role of building a generation able to life and work in this era. Various educational studies such as Abdulkader (2014), Alnabawy and Galal (2016) and Kayang \& Msiska (2016) results confirmed existence of deficiencies in teachers' $21^{\text {st }}$ century skills and need for developing training programs to ensure teachers having these skills and functionally mastering these skills.

Although it is necessary to work on teacher acquiring 21 st century skills and great interest in it, there no educational study - as far as researcher knows - concerned with university staff members training needs in light of these skills. Even

though some educational studies have concerned with these skills in terms of analytical descriptive of study, evaluating some syllabuses in light of these skills, or present a proposed scenario for development these skills by integrating them with a school curricula.

\section{Study problem:}

The process of identifying training needs is the first and core step of from which training process starts, as it declare training goals and help in in good planning for training programs. Success in this step require determining these needs accurately to achieve its purpose in light of objective indicators and bases effectively contribute in developing trainees' skills and abilities.

Al-Azhar University
currently witness
comprehensive development in
all fields, especially in organizational field. This development accompanied by increase in university staff members with great attention for preparing and qualifying them. University staff members' professional development is the way to reform and develop higher 
education system to cope with changes and challenges currently taking place in society. These challenges which have reflection on educational system in terms of its role in provide educational opportunities compatible with the labor market and sustainable development requirements, which all

advanced countries abide with to achieve its aims through 21 st century skills.

The fact that university staff member is of the important pillars of university success in achieving its aims and an important indicator to $\mathrm{m}$ aintain awareness and quality control

of educational outcomes.

University staff member coping with various developments to be able to continue performing his roles in desired manner requires specifying his training that keep pace with professional roles.

Starting from importance of university staff members possess 21 st century skills and its effect in work development and raising education quality and university outputs. Researcher worked through the current study to contribute to achieve this by identifying the training needs of university staff members at faculty of physical education, Al-Azhar University, according to their tasks and their coping with global development in the field of university education and in light of 21 st century skills. To achieve this, current study trying to answer the following queries:

1. What are training needs of university staff members at faculty of physical education, Al-Azhar University in light of 21st century skills?

2. Is there statistically significant differen ces between training needs of staff members at faculty of physical education, Al-Azhar University in light of 21st century skills in accordance with scientific qualification and scientific department variables?

Study importance:

Current study importance is due of the following:

1- Draw the attention of educators concerned with higher education development and improvement to the recent trends that should be considered when designing the training programs for university staff members.

2- Alignment with future visions for higher education system development scenarios in all its elements, which are calling for attention with $21 \mathrm{st}$ century skills to achieve this development. 
3- Enriching educational

library as it considered one of the first educational studies concerned with identifying training needs of staff members at faculty of physical education, Al-Azhar University in light of 21st century skills.

Study objectives:

The present study aimed to achieve the following: 1. Determine training needs of university staff members at faculty of physical education, Al-Azhar University in light of 21 st century skills.

2. Identify statistics differences between training needs of staff members at faculty of physical education, Al-Azhar University in light of 21 st century skills in accordance with scientific qualification and scientific department variables.

Study hypotheses:

1. There are no statisticall

y significant

differences between training needs of staff members at faculty of physical education, Al-Azhar University in light of 21 st century skills in accordance with scientific qualification variable.
2. There are no statisticall y significant differences between training needs of staff members at faculty of physical education, Al-Azhar University in light of 21st century skills in accordance with scientific department variable.

\section{Study procedures:}

\section{Study methodology:}

The study used descriptive method as it suits study nature and objectives, using questionnaire as data collecting tool.

Study population and sample:

The

population consisted of

staff members at Faculty of Physical Education, Al-Azhar University, they are all staff members during study period in second term of the academic year 2017/2018 according to official statistics for staff members management in Faculty of Physical Education, Al-Azhar University . Study sample was (25) staff members at Faculty of Physical Education, Al-Azhar

University who responded by answering the qu estionnaire items, they represent $(58.14 \%)$ of 


\section{5}

study society, the following tab according to study variables : le shows sample individuals

Table (1) Study sample distribution according to study variables

\begin{tabular}{c|c|c|c}
\hline \hline Variable & Categories & Number & Percentage \\
\hline \hline Educational Qualification & PhD & 14 & $44 \%$ \\
\cline { 2 - 4 } Scientific Section & Master & 11 & $56 \%$ \\
\cline { 2 - 4 } & $\begin{array}{c}\text { Grating department } \\
\text { Training, Management) }\end{array}$ & 16 & $64 \%$ \\
\cline { 2 - 4 } & $\begin{array}{c}\text { Non granting } \\
\text { departments } \\
\text { ( Health Sciences }, \\
\text { movement } \\
\text { science, Psychology) }\end{array}$ & 9 & $36 \%$ \\
\hline \hline & \begin{tabular}{c} 
Total \\
\hline
\end{tabular} \\
\hline \hline
\end{tabular}

\section{Study tool:}

To achieve study objective, a questionnaire developed for data collection and identify training need of staff members at faculty of physical education, $\mathrm{Al}$ - Azhar University, in light of 21st century skills. This done through analysis of some documents related to study subject, such as (AlDahshan \& Badran, 2001; Mahmoud, 2001; Bashir, 2004; Teima, R. \& Albandary, 2004; Mahmoud, 2009; Ishaq, 2011; Trilling \& Fadel, 2009; Abdullah \& Abo Elfadl, 2014 \& Sadiq, 2014) aiming to develop the study tool and

\section{Table (2)}

wording of its measurable phrases. In addition to conduct some personal interviews with some university staff members at faculty of physical education, Al-Azhar University, in order to benefit from their experience in preparing the questionnaire. Accordingly,

questionnaire developed consisting of (59) phrases that cover all aspects of training needs in light of $21 \mathrm{st}$ century skills, distributed to seven basic aspects represent 21st skills, following table illustrates this: 
Questionnaire aspects and training needs number in light of it

\begin{tabular}{|c|c|c|c|}
\hline S.No & questionnaire aspects & $\begin{array}{c}\text { number of } \\
\text { training needs }\end{array}$ & $\begin{array}{c}\text { Relative } \\
\text { weight }\end{array}$ \\
\hline 1 & Innovation and creativity skill. & 10 & $16,95 \%$ \\
\hline 2 & $\begin{array}{l}\text { Critical thinking and problem } \\
\text { solving skill. }\end{array}$ & 8 & $13.56 \%$ \\
\hline 3 & $\begin{array}{l}\text { Information and } \\
\text { technological culture skill. }\end{array}$ & 13 & $22,03 \%$ \\
\hline 4 & Communication skill. & 9 & $15,25 \%$ \\
\hline 5 & $\begin{array}{l}\text { Cooperation, leadership and } \\
\text { responsibility skill. }\end{array}$ & 6 & $10,17 \%$ \\
\hline 6 & $\begin{array}{l}\text { Flexibility and } \\
\text { adaptability skill. }\end{array}$ & 7 & $11,86 \%$ \\
\hline 7 & \multirow[t]{2}{*}{$\begin{array}{l}\text { Self- directing and productivity } \\
\text { skill. }\end{array}$} & 6 & $10,17 \%$ \\
\hline Total & & 59 & $100 \%$ \\
\hline \multicolumn{2}{|c|}{$\begin{array}{l}\text { Each phrase measured } \\
\text { using 5-point Likert scale to } \\
\text { declare the training need } \\
\text { degree as following: very high } \\
\text { (5), high (4), average ( } 3) \text {, low } \\
\text { (2), and very low (1), and to } \\
\text { determine the degree } \\
\text { of training need Closed } \\
\text { Questionnaire form adopted, } \\
\text { which assign the average (3.5- } \\
\text { 5) to (high need), (2.5-3.49) } \\
\text { to (average need), and (1- } \\
\text { 2,49) to (low need). } \\
\text { Questionnaire reliability and } \\
\text { validity: } \\
\text { Questionnaire validity: }\end{array}$} & \multicolumn{2}{|c|}{$\begin{array}{l}\text { from staff members at faculties } \\
\text { of physical education. That is } \\
\text { to ensure validity of } \\
\text { the questionnaire as data } \\
\text { collection tool, phrasing proper } \\
\text { language, and to identify } \\
\text { proposed training } \\
\text { needs appropriateness for each } \\
\text { skill of 21st century } \\
\text { skills. Based on opinions and } \\
\text { observations, researcher carried } \\
\text { out appropriate adjustments } \\
\text { arrived to the questionnaire in } \\
\text { its final form (appendix 1) } \\
\text { Questionnaire reliability: } \\
\text { Questionnaire }\end{array}$} \\
\hline $\begin{array}{l}\text { confirn } \\
\text { validity } \\
\text { experts }\end{array}$ & $\begin{array}{l}\text { Questionnaire validity } \\
\text { med using content } \\
\text { y by presenting it to (10) } \\
\text { s and specialists }\end{array}$ & $\begin{array}{l}\text { reliability confirm } \\
\text { (Test-Retest) } \\
\text { presenting the quest } \\
\text { sample of } 10 \text { univ }\end{array}$ & $\begin{array}{l}\text { ed using } \\
\text { Through } \\
\text { ionnaire to } \\
\text { ersity staff }\end{array}$ \\
\hline
\end{tabular}


members from the study population and not included in study sample with 15

days interval time, results illustrated in the following table:

Table (3)

Correlation coefficients between first and second administration of training needs in light of 21 st century skills questionnaire $(n=10)$

\begin{tabular}{|c|c|c|c|c|c|c|c|}
\hline \multirow[t]{2}{*}{$\begin{array}{l}\text { S. } \\
\text { No }\end{array}$} & \multirow[t]{2}{*}{ training needs } & \multicolumn{2}{|c|}{$\begin{array}{c}1^{\text {st }} \\
\text { administration }\end{array}$} & \multicolumn{2}{|c|}{$\begin{array}{c}\text { 2nd } \\
\text { administration }\end{array}$} & \multirow[t]{2}{*}{$\begin{array}{c}\mathrm{R} \\
\text { value }\end{array}$} & \multirow[t]{2}{*}{$\begin{array}{l}\text { Signf. } \\
\text { level }\end{array}$} \\
\hline & & Mean & SD & Mean & SD & & \\
\hline \multicolumn{8}{|c|}{ First: Innovation and creativity skill: } \\
\hline 1 & $\begin{array}{l}\text { Creative planning, implementation and } \\
\text { evaluation based on effective teaching. }\end{array}$ & 4.42 & 1.22 & 4.57 & 1.15 & 0.90 & SIG. \\
\hline 2 & $\begin{array}{l}\text { Time management to achieve desired } \\
\text { goals in the specified time her. }\end{array}$ & 4.12 & 0.84 & 4.53 & 1.00 & 0.89 & SIG. \\
\hline 3 & $\begin{array}{l}\text { Selecting teaching } \\
\text { strategies that contribute to developing } \\
\text { students' creative thinking. }\end{array}$ & 4.51 & 1.64 & 4.59 & 0.95 & 0.85 & SIG. \\
\hline 4 & $\begin{array}{l}\text { Developing high-level thinking skills } \\
\text { among students through different. } \\
\text { activities }\end{array}$ & 4.25 & 0.96 & 4.48 & 1.41 & 0.96 & SIG. \\
\hline 5 & $\begin{array}{l}\text { Using current events and life situation } \\
\text { to deliver educational content to } \\
\text { students. }\end{array}$ & 4.31 & 1.06 & 4.71 & 1.22 & 0.81 & SIG. \\
\hline 6 & Using modern teaching strategies. & 11.4 & 0.48 & 16.4 & 1.12 & 0.91 & SIG. \\
\hline 7 & $\begin{array}{l}\text { Designing diverse enrichment situation } \\
\text { and activities to develop students } \\
\text { multiple intelligences and life skills. }\end{array}$ & 4.59 & 1.25 & 4.37 & 1.03 & 0.89 & SIG. \\
\hline 8 & $\begin{array}{l}\text { Using more than one the teaching } \\
\text { strategy in same lecture. }\end{array}$ & 4.28 & 1.26 & 4.33 & 1.48 & 0.85 & SIG. \\
\hline 9 & $\begin{array}{l}\text { Producing and directing objective } \\
\text { educational activities in variety of } \\
\text { creative. }\end{array}$ & 4.11 & 1.04 & 4.42 & 0.92 & 0.87 & SIG. \\
\hline 10 & $\begin{array}{l}\text { Designing and functionally using } \\
\text { different evaluation tools. }\end{array}$ & 4.16 & 1.12 & 4.43 & 1.05 & 0.92 & SIG. \\
\hline \multicolumn{8}{|c|}{ Second : Critical thinking and problem solving skill: } \\
\hline 1 & $\begin{array}{l}\text { Using different thinking methods in } \\
\text { educational situations. }\end{array}$ & 4.41 & 0.89 & 4.64 & 0.71 & 0.91 & SIG. \\
\hline 2 & $\begin{array}{l}\text { Producing creative problem solutions } \\
\text { during work. }\end{array}$ & 4.51 & 0.71 & 4.57 & 0.91 & 0.89 & SIG. \\
\hline 3 & $\begin{array}{l}\text { Solving problems in classrooms in } \\
\text { appropriate methods. }\end{array}$ & 4.42 & 1.45 & 4.48 & 1.48 & 0.93 & SIG. \\
\hline 4 & $\begin{array}{l}\text { Scientific positive critics though } \\
\text { introducing community issues for } \\
\text { dialogue and discussion. }\end{array}$ & 4.01 & 1.57 & 4.21 & 1.09 & 0.87 & SIG. \\
\hline
\end{tabular}

Follow Table (3) 
Correlation coefficients between first and second administration of training needs in light of 21st century skills questionnaire $(n=10)$

\begin{tabular}{|c|c|c|c|c|c|c|c|}
\hline \multirow[t]{2}{*}{$\begin{array}{c}\text { S. } \\
\text { No }\end{array}$} & \multirow[t]{2}{*}{ training needs } & \multicolumn{2}{|c|}{$\begin{array}{c}1^{\text {st }} \\
\text { administration }\end{array}$} & \multicolumn{2}{|c|}{$\begin{array}{c}\text { 2nd } \\
\text { administration }\end{array}$} & \multirow[t]{2}{*}{$\begin{array}{c}\mathrm{R} \\
\text { value }\end{array}$} & \multirow[t]{2}{*}{$\begin{array}{l}\text { Signf. } \\
\text { level }\end{array}$} \\
\hline & & Mean & SD & Mean & SD & & \\
\hline$\overline{5}$ & $\begin{array}{l}\text { Getting right information from their } \\
\text { sources for judging in light of it. }\end{array}$ & 4.24 & 0.67 & 4.31 & 1.12 & 0.91 & SIG. \\
\hline 6 & $\begin{array}{l}\text { Correcting errors and lack } \\
\text { of knowledge in creative methods. }\end{array}$ & 4.40 & 1.07 & 4.61 & 1.01 & 0.85 & SIG. \\
\hline 7 & $\begin{array}{l}\text { Using self-evaluating tools and } \\
\text { the issuing objective judgments } \\
\text { through it. }\end{array}$ & 4.28 & 1.26 & 4.42 & 1.38 & 0.91 & SIG. \\
\hline 8 & $\begin{array}{l}\text { Using dialogue and discussion methods } \\
\text { to present education content problems, } \\
\text { and propose appropriate solutions }\end{array}$ & 4.42 & 1.45 & 4.61 & 1.48 & 0.87 & SIG. \\
\hline \multicolumn{8}{|c|}{ Third : Information and technological culture skill: } \\
\hline 1 & $\begin{array}{l}\text { Knowing websites that release the new } \\
\text { in specialty. }\end{array}$ & 4.31 & 0.89 & 4.54 & 1.04 & 0.89 & SIG. \\
\hline 2 & $\begin{array}{l}\text { Knowing information searching and } \\
\text { investigation mechanism. }\end{array}$ & 4.16 & 0.97 & 4.50 & 0.71 & 0.95 & SIG. \\
\hline 3 & $\begin{array}{l}\text { Documentation of information } \\
\text { obtained from various sources }\end{array}$ & 4.24 & 0.67 & 4.31 & 1.09 & 0.91 & SIG. \\
\hline 4 & $\begin{array}{l}\text { Knowing scientific criteria on which } \\
\text { information validated, }\end{array}$ & 4.30 & 0.57 & 4.42 & 0.67 & 0.93 & SIG. \\
\hline 5 & $\begin{array}{l}\text { Constructive criticism of } \\
\text { media messages and adapt it to serve } \\
\text { the educational content }\end{array}$ & 4.27 & 0.88 & 4.44 & 1.09 & 0.90 & SIG. \\
\hline 6 & $\begin{array}{l}\text { Using media news in teaching skills } \\
\text { in creative ways }\end{array}$ & 4.40 & 1.07 & 4.61 & 1.01 & 0.91 & SIG. \\
\hline 7. & $\begin{array}{l}\text { Activating the role } \\
\text { of educational media in discussing } \\
\text { community issues }\end{array}$ & 4.28 & 1.26 & 4.32 & 1.48 & 0.89 & SIG. \\
\hline 8 & $\begin{array}{l}\text { Utilization of information and } \\
\text { knowledge in educational content in } \\
\text { a functional manner. }\end{array}$ & 4.12 & 0.84 & 4.33 & 1.01 & 0.91 & SIG. \\
\hline 9 & $\begin{array}{l}\text { Using educational media in developing } \\
\text { students' information culture. }\end{array}$ & 4.42 & 1.22 & 4.57 & 1.15 & 0.90 & SIG. \\
\hline 10 & $\begin{array}{l}\text { Knowing technical and educational } \\
\text { standards for electronic content design. }\end{array}$ & 4.25 & 0.96 & 4.48 & 1.01 & 0.94 & SIG. \\
\hline 11 & $\begin{array}{l}\text { Design and use of } \\
\text { computer programs in the service of } \\
\text { educational content. }\end{array}$ & 4.50 & 1.64 & 4.59 & 1.22 & 0.89 & SIG. \\
\hline 12 & $\begin{array}{l}\text { Participate actively in forums related } \\
\text { to specialization. }\end{array}$ & 4.36 & 0.52 & 4.53 & 0.75 & 0.87 & SIG. \\
\hline 13 & $\begin{array}{l}\text { Using different web tools in } \\
\text { educational process. }\end{array}$ & 4.59 & 1.25 & 4.60 & 1.13 & 0.89 & SIG. \\
\hline
\end{tabular}

Follow Table (3) 
Correlation coefficients between first and second administration of training needs in light of 21st century skills questionnaire $(n=10)$

\begin{tabular}{|c|c|c|c|c|c|c|c|}
\hline \multirow[t]{2}{*}{$\begin{array}{l}\text { S. } \\
\text { No }\end{array}$} & \multirow[t]{2}{*}{ training needs } & \multicolumn{2}{|c|}{$\begin{array}{c}1^{\text {st }} \\
\text { administration }\end{array}$} & \multicolumn{2}{|c|}{$\begin{array}{c}\text { 2nd } \\
\text { administration }\end{array}$} & \multirow[t]{2}{*}{$\begin{array}{c}\mathrm{R} \\
\text { value }\end{array}$} & \multirow[t]{2}{*}{$\begin{array}{l}\begin{array}{l}\text { Signf. } \\
\text { level }\end{array} \\
\end{array}$} \\
\hline & & Mean & SD & Mean & SD & & \\
\hline \multicolumn{8}{|c|}{ Fourth : Communication skill: } \\
\hline 1 & $\begin{array}{l}\text { Organization of enrichment scientific } \\
\text { material and present it in logical } \\
\text { manner. }\end{array}$ & 4.31 & 1.06 & 4.42 & 1.22 & 0.83 & SIG. \\
\hline 2 & $\begin{array}{l}\text { Support and promote students' } \\
\text { motivation. }\end{array}$ & 4.01 & 1.57 & 4.35 & 1.10 & 0.89 & SIG. \\
\hline 3 & $\begin{array}{l}\text { Direct students towards lifelong } \\
\text { learning necessity. }\end{array}$ & 4.15 & 0.42 & 4.33 & 0.92 & 0.91 & SIG. \\
\hline 4 & $\begin{array}{l}\text { Knowing positive dialogue bases when } \\
\text { discussing different issues. }\end{array}$ & 4.42 & 1.45 & 4.51 & 1.48 & 0.93 & SIG. \\
\hline 5 & $\begin{array}{l}\text { Designing and implementing dialogue } \\
\text { and discussion scenario and not leave it } \\
\text { to chance. }\end{array}$ & 4.50 & 0.71 & 4.71 & 0.94 & 0.90 & SIG. \\
\hline 6 & $\begin{array}{l}\text { Contributing to achieve students' } \\
\text { intellectual safety. }\end{array}$ & 4.12 & 0.84 & 4.35 & 1.12 & 0.92 & SIG. \\
\hline 7 & $\begin{array}{l}\text { Using more than one } \\
\text { communication method to express } \\
\text { views and ideas. }\end{array}$ & 4.36 & 0.57 & 4.61 & 0.57 & 0.87 & SIG. \\
\hline 8 & $\begin{array}{l}\text { Express opinions effectively and } \\
\text { fluently in front of all. }\end{array}$ & 4.16 & 0.97 & 4.40 & 0.82 & 0.95 & SIG. \\
\hline 9 & $\begin{array}{l}\text { Providing safe and support climate for } \\
\text { creative learning. }\end{array}$ & 4.40 & 1.07 & 4.61 & 1.01 & 0.85 & SIG. \\
\hline \multicolumn{8}{|c|}{ Fifth : Cooperation, leadership and responsibility skill: } \\
\hline 1 & $\begin{array}{l}\text { Knowing educational standards for } \\
\text { using cooperative strategies during } \\
\text { teaching. }\end{array}$ & 4.31 & 1.01 & 4.42 & 1.12 & 0.84 & SIG. \\
\hline 2 & $\begin{array}{l}\text { Carry out difficult tasks that need to } \\
\text { more creative thinking in } \\
\text { accomplishing it }\end{array}$ & 4.25 & 0.94 & 4.48 & 1.02 & 0.89 & SIG. \\
\hline 3 & $\begin{array}{l}\text { Exercise leadership team through } \\
\text { organization of collective work. }\end{array}$ & 4.28 & 1.26 & 4.38 & 1.29 & 0.95 & SIG. \\
\hline 4 & $\begin{array}{l}\text { Knowing profession ethics of and work } \\
\text { to develop them in order to achieve } \\
\text { desired learning outcomes. }\end{array}$ & 4.41 & 0.89 & 4.64 & 0.71 & 0.91 & SIG. \\
\hline 5 & $\begin{array}{l}\text { Knowing leadership skills through } \\
\text { seminars with specialists. }\end{array}$ & 4.30 & 0.57 & 4.41 & 0.78 & 0.93 & SIG. \\
\hline 6 & $\begin{array}{l}\text { Knowing partnership methods with } \\
\text { external community organizations to } \\
\text { develop educational process. }\end{array}$ & 4.01 & 1.15 & 4.31 & 1.02 & 0.90 & SIG. \\
\hline
\end{tabular}

Follow Table (3) 
Correlation coefficients between first and second administration of training needs in light of 21st century skills questionnaire $(n=10)$

\begin{tabular}{|c|c|c|c|c|c|c|c|}
\hline \multirow[t]{2}{*}{$\begin{array}{l}\text { S. } \\
\text { No }\end{array}$} & \multirow[t]{2}{*}{ training needs } & \multicolumn{2}{|c|}{$\begin{array}{c}1^{\text {st }} \\
\text { administration }\end{array}$} & \multicolumn{2}{|c|}{$\begin{array}{c}\text { 2nd } \\
\text { administration }\end{array}$} & \multirow[t]{2}{*}{$\begin{array}{c}\mathrm{R} \\
\text { value }\end{array}$} & \multirow[t]{2}{*}{$\begin{array}{l}\text { Signf. } \\
\text { level }\end{array}$} \\
\hline & & Mean & SD & Mean & SD & & \\
\hline \multicolumn{8}{|c|}{ Sixth : Flexibility and adaptability skill: } \\
\hline 1 & $\begin{array}{l}\text { Dealing with modern teaching } \\
\text { aids in teaching. }\end{array}$ & 4.24 & 0.67 & 4.31 & 1.09 & 0.91 & SIG. \\
\hline 2 & $\begin{array}{l}\text { Knowing regulations and } \\
\text { laws that control educational process. }\end{array}$ & 4.28 & 1.26 & 4.32 & 1.48 & 0.89 & SIG. \\
\hline 3 & $\begin{array}{l}\text { Develop flexible plans on sound } \\
\text { scientific bases. }\end{array}$ & 4.36 & 0.58 & 4.53 & 0.95 & 0.86 & SIG. \\
\hline 4 & $\begin{array}{l}\text { Modification of existing plans based } \\
\text { on hypothetical changes }\end{array}$ & 4.31 & 1.16 & 4.38 & 1.24 & 0.94 & SIG. \\
\hline 5 & $\begin{array}{l}\text { Awareness of new curricula } \\
\text { importance and achievement } \\
\text { mechanisms. }\end{array}$ & 4.15 & 0.42 & 4.33 & 0.82 & 0.92 & SIG. \\
\hline 6 & $\begin{array}{l}\text { Provide feedback to } \\
\text { modify shortcomings when } \\
\text { teaching students. }\end{array}$ & 4.12 & 0.85 & 4.42 & 1.12 & 0.89 & SIG. \\
\hline 7 & $\begin{array}{l}\text { Provide immediate reinforcement and } \\
\text { support for correct learners' responses. }\end{array}$ & 4.28 & 1.18 & 4.42 & 1.31 & 0.85 & SIG. \\
\hline \multicolumn{8}{|c|}{ Seventh : Self -directing and productivity skill: } \\
\hline 1 & $\begin{array}{l}\text { Identify strengths and weaknesses } \\
\text { through the analysis of self-evaluation } \\
\text { tools. }\end{array}$ & 4.09 & 1.12 & 4.28 & 1.07 & 0.90 & SIG. \\
\hline 2 & $\begin{array}{l}\text { Using available environmental } \\
\text { resources in implementation of } \\
\text { students' activities. }\end{array}$ & 4.15 & 0.78 & 4.38 & 0.82 & 0.92 & SIG. \\
\hline 3 & $\begin{array}{l}\text { Knowing student } \\
\text { projects that contribute in achieving } \\
\text { curricula goals. }\end{array}$ & 4.41 & 0.98 & 4.46 & 1.07 & 0.89 & SIG. \\
\hline 4 & $\begin{array}{l}\text { Knowing objective ways to use self- } \\
\text { evaluating tools to achieve its goals. }\end{array}$ & 4.11 & 0.84 & 4.32 & 0.71 & 0.91 & SIG. \\
\hline 5 & $\begin{array}{l}\text { Organizing productive projects that suit } \\
\text { students' interests. }\end{array}$ & 4.42 & 1.54 & 4.52 & 1.52 & 0.95 & SIG. \\
\hline 6 & $\begin{array}{l}\text { Knowing analysis mechanism of } \\
\text { education reality forms and benefit } \\
\text { from it }\end{array}$ & 4.31 & 1.16 & 4.42 & 1.09 & 0.92 & SIG.. \\
\hline
\end{tabular}

(r) significant at (0.05) level $=0.63$

Table (3) results reveal statistically significant correlation between first and second application as correlation coefficient ranged

between (0.81 and 0.96), the matter which confirm questionnaire reliability and in turning results from applicable study tool. 
Questionnaire

final form administration:

Questionnaire final

form consisting of (59)

training needs distributed on seven aspects representing 21st century skills administrated on a sample (25) staff members at faculty of physical education, Al- Azhar University.

Questionnaire administration carried out during the period 10-14/12/2017, researcher

explained study purpose and clarify training needs in each aspect and response instructions, and then data collected and prepared to conduct appropriate statistical process.

\section{Statistical Process:}

Researcher

used

SPSS statistical program to analyze data to arrive to study results, using following statistical methods: mean, standard deviation, percentage, Alpha Cronbach coefficient, and $\mathrm{T}$ test. All date analyzed in 0.05 significance level.

\section{Results and discussion:}

\section{I- First query results:}

Each aspect results will be presented separately, while interpretation and discussion will be for questionnaire in overall:

\section{First aspect: Innovation and} creativity skill:

Table (4)

Study sample responses in innovation and creativity aspect skill $(n=25)$

\begin{tabular}{l|l|c|c|c|c|c}
\hline \hline $\begin{array}{l}\text { S. } \\
\text { No }\end{array}$ & training needs & Mean & SD & $\begin{array}{c}\text { Relative } \\
\text { weight }\end{array}$ & $\begin{array}{c}\text { need } \\
\text { degree }\end{array}$ & Rank \\
\hline \hline 1 & $\begin{array}{l}\text { Creative } \\
\text { implementation planning, } \\
\text { evaluation based on effective } \\
\text { teaching. }\end{array}$ & 4.27 & 1.15 & 85.4 & High & 3 \\
\hline 2 & $\begin{array}{l}\text { Time management to achieve } \\
\text { desired goals in the specified } \\
\text { time her. }\end{array}$ & 4.35 & 1.24 & 87.0 & High & 2 \\
\hline 3 & $\begin{array}{l}\text { Selecting teaching } \\
\text { strategies that contribute to } \\
\text { developing students' creative } \\
\text { thinking. }\end{array}$ & 4.69 & 0.94 & 93.8 & High & 1 \\
\hline 4 & $\begin{array}{l}\text { Developing high-level } \\
\text { thinking skills among students } \\
\text { through different. Activities }\end{array}$ & 4.16 & 1.02 & 83.2 & High & 5 \\
\hline \hline
\end{tabular}

Follow Table (4)

Assiut Journal For Sport Science Arts 
Study sample responses in innovation and creativity aspect skill $(n=25)$

\begin{tabular}{c|l|c|c|c|c|c}
\hline \hline $\begin{array}{c}\text { S. } \\
\text { No }\end{array}$ & training needs & Mean & SD & $\begin{array}{c}\text { Relative } \\
\text { weight }\end{array}$ & $\begin{array}{c}\text { need } \\
\text { degree }\end{array}$ & Rank \\
\hline \hline 5 & $\begin{array}{l}\text { Using current events and life } \\
\text { situation to deliver educational } \\
\text { content to students. }\end{array}$ & 4.02 & 1.11 & 80.4 & High & 8 \\
\hline 6 & $\begin{array}{l}\text { Using } \\
\text { modern teaching strategies. }\end{array}$ & 4.24 & 1.19 & 76,8 & High & 4 \\
\hline 7 & $\begin{array}{l}\text { Designing diverse enrichment } \\
\text { situation and activities to } \\
\text { develop students multiple } \\
\text { intelligences and life skills. }\end{array}$ & 3.89 & 1.17 & 77.8 & High & 9 \\
\hline 8 & $\begin{array}{l}\text { Using more than one the teaching } \\
\text { strategy in same lecture. }\end{array}$ & 4.11 & 0.98 & 82.2 & High & 7 \\
\hline 9 & $\begin{array}{l}\text { Producing and dircting objective } \\
\text { educational activities in variety of } \\
\text { creative. }\end{array}$ & 4.15 & 1.04 & 83.0 & High & 6 \\
\hline 10 & $\begin{array}{l}\text { Designing and functionally using } \\
\text { different evaluation tools. }\end{array}$ & 3.84 & 1.17 & 84.8 & High & 10 \\
\hline \multicolumn{7}{l}{$\begin{array}{l}\text { General average } \\
\text { Table (4) results reveal }\end{array}$} \\
\hline \hline
\end{tabular}

Table (5)

Study sample responses in Critical thinking and problem solving skill $(\mathbf{n}=25)$

\begin{tabular}{c|l|c|c|c|c|c}
\hline \hline $\begin{array}{c}\text { S. } \\
\text { No }\end{array}$ & training needs & Mean & SD & $\begin{array}{c}\text { Relative } \\
\text { weight }\end{array}$ & $\begin{array}{c}\text { need } \\
\text { degree }\end{array}$ & Rank \\
\hline \hline 1 & $\begin{array}{l}\text { Using different thinking } \\
\text { methods in educational } \\
\text { situations. }\end{array}$ & 4.37 & 1.15 & 87.4 & High & 4 \\
\hline 2 & $\begin{array}{l}\text { Producing creative problem } \\
\text { solutions during work. }\end{array}$ & 4.29 & 0.89 & 85.8 & High & 5 \\
\hline 3 & $\begin{array}{l}\text { Solving problems in } \\
\text { classrooms in } \\
\text { appropriate methods. }\end{array}$ & 4.38 & 1.11 & 87.6 & High & 3 \\
\hline \hline
\end{tabular}

Follow Table (5)

Assiut Journal For Sport Science Arts 
Study sample responses in Critical thinking and problem solving

skill $(n=25)$

\begin{tabular}{c|l|c|c|c|c|c}
\hline \hline $\begin{array}{c}\text { S. } \\
\text { No }\end{array}$ & training needs & Mean & SD & $\begin{array}{c}\text { Relative } \\
\text { weight }\end{array}$ & $\begin{array}{c}\text { need } \\
\text { degree }\end{array}$ & Rank \\
\hline \hline 4 & $\begin{array}{l}\text { Scientific positive } \\
\text { critics though } \\
\text { introducing community } \\
\text { issues for dialogue and } \\
\text { discussion. }\end{array}$ & 4.65 & 1.02 & 93.0 & High & 1 \\
\hline 5 & $\begin{array}{l}\text { Getting right } \\
\text { information from their } \\
\text { sources for judging in } \\
\text { light of it. }\end{array}$ & 4.44 & 0.98 & 88.8 & High & 2 \\
\hline 6 & $\begin{array}{l}\text { Correcting errors and } \\
\text { lack of knowledge } \\
\text { in creative methods. }\end{array}$ & 4.12 & 1.04 & 82.4 & High & 6 \\
\hline 7 & $\begin{array}{l}\text { Using self-evaluating } \\
\text { tools and the issuing } \\
\text { objective judgments } \\
\text { through it. }\end{array}$ & 3.94 & 0.97 & 78.8 & High & 8 \\
\hline 8 & $\begin{array}{l}\text { Using dialogue and } \\
\text { discussion methods to } \\
\text { present } \\
\text { education content } \\
\text { problems, and propose } \\
\text { appropriate solutions }\end{array}$ & 4.11 & 0.93 & 82.2 & High & 7 \\
\hline \hline
\end{tabular}

Table (5) results reveal that training needs degree for Critical thinking and problem solving skill aspect is high with mean of (4.29). The greatest need was need (4) "Scientific positive critics though introducing community issues for dialogue and discussion." with high degree

\section{Table (6)}

and mean of (4.65). The lowest need was need (7) "Using selfevaluating tools and the issuing objective judgments through it.." with high degree and mean of (3.94).

Third aspect: Information and technological culture skill: 
Study sample responses in Information and technological culture

skill $(\mathbf{n}=25)$

\begin{tabular}{|c|c|c|c|c|c|c|}
\hline $\begin{array}{l}\text { S. } \\
\text { No }\end{array}$ & training needs & Mean & SD & $\begin{array}{l}\text { Relative } \\
\text { weight }\end{array}$ & $\begin{array}{l}\text { need } \\
\text { degree }\end{array}$ & Rank \\
\hline $\bar{~} 1$ & $\begin{array}{l}\text { Knowing websites that release } \\
\text { the new in specialty. }\end{array}$ & 4.68 & 1.14 & 93.6 & High & 8 \\
\hline 2 & $\begin{array}{l}\text { Knowing information searching } \\
\text { and investigation mechanism. }\end{array}$ & 4.77 & 0.97 & 95.4 & High & 3 \\
\hline 3 & $\begin{array}{l}\text { Documentation of information } \\
\text { obtained from various sources }\end{array}$ & 4.43 & 0.84 & 88.6 & High & 13 \\
\hline 4 & $\begin{array}{lr}\text { Knowing } & \text { scientific criteria } \\
\text { on which } & \text { information } \\
\text { validated, } & \\
\end{array}$ & 4.76 & 1.01 & 95.2 & High & 4 \\
\hline 5 & $\begin{array}{l}\text { Constructive criticism of } \\
\text { media messages and adapt it to } \\
\text { serve the educational content }\end{array}$ & 4.65 & 0.94 & 93.0 & High & 9 \\
\hline 6 & $\begin{array}{l}\text { Using media news in teaching } \\
\text { skills in creative ways }\end{array}$ & 4.78 & .87 & 95.6 & High & 2 \\
\hline 7 & $\begin{array}{l}\text { Activating the role } \\
\text { of educational media in } \\
\text { discussing community issues }\end{array}$ & 4.47 & 1.04 & 89.4 & High & 12 \\
\hline 8 & $\begin{array}{l}\text { Utilization of information and } \\
\text { knowledge } \\
\text { in educational content in } \\
\text { a functional manner. }\end{array}$ & 4.71 & $\begin{array}{c}0 \\
\text { and } \\
98\end{array}$ & 94.2 & High & 6 \\
\hline 9 & $\begin{array}{l}\text { Using educational media in } \\
\text { developing } \\
\text { information culture. }\end{array}$ & 4.75 & 1.17 & 95,0 & High & 5 \\
\hline 10 & $\begin{array}{lcr}\text { Knowing } & \text { technical and } \\
\text { educational } & \text { standards } \\
\text { for electronic content design. }\end{array}$ & 4.59 & 1.09 & 91,8 & High & 11 \\
\hline 11 & $\begin{array}{l}\text { Design and use of } \\
\text { computer programs in the } \\
\text { service of educational content. }\end{array}$ & 4.85 & 0.89 & 97.0 & High & 1 \\
\hline 12 & $\begin{array}{l}\text { Participate actively in forums } \\
\text { related to specialization. }\end{array}$ & 4.64 & 0.95 & 92.8 & High & 10 \\
\hline 13 & $\begin{array}{l}\text { Using different web tools in } \\
\text { educational process. }\end{array}$ & 4.69 & 1.07 & 93.8 & High & 7 \\
\hline \multicolumn{2}{|c|}{ General average } & 4.67 & 0.89 & 93.4 & High & \\
\hline
\end{tabular}

Table (6) results reveal

that training needs degree for

Information and technological

culture skill aspect is high with 
mean of (4.67). The greatest need was need (11) "Design and use of computer programs in the service of educational content" with high degree and mean of (4.85). The lowest need was need

"Documentation of information obtained from various sources" with high degree and mean of (4.43).

\section{Forth}

Table (7)

Study sample responses in communication skill $(\mathbf{n}=\mathbf{2 5})$

\begin{tabular}{l|l|c|c|c|c|c}
\hline \hline $\begin{array}{l}\text { S. } \\
\text { No }\end{array}$ & training needs & Mean & SD & $\begin{array}{l}\text { Relative } \\
\text { weight }\end{array}$ & $\begin{array}{l}\text { need } \\
\text { degree }\end{array}$ & Rank \\
\hline \hline 1 & $\begin{array}{l}\text { Organization of enrichment } \\
\text { scientific material and } \\
\text { present it in logical manner. }\end{array}$ & 3.67 & 0.96 & 73.4 & High & 1 \\
\hline 2 & $\begin{array}{l}\text { Support and promote } \\
\text { students' motivation. }\end{array}$ & 3.53 & 1.04 & 70.6 & High & 4 \\
\hline 3 & $\begin{array}{l}\text { Direct students towards } \\
\text { lifelong learning necessity. }\end{array}$ & 3.42 & 1.07 & 68.4 & average & 6 \\
\hline 4 & $\begin{array}{l}\text { Knowing } \\
\text { positive dialogue bases } \\
\text { when discussing different } \\
\text { issues. }\end{array}$ & 3.47 & 0.81 & 69.4 & average & 5 \\
\hline 5 & $\begin{array}{l}\text { Designing and } \\
\text { implementing dialogue and } \\
\text { discussion scenario and not } \\
\text { leave it to chance. }\end{array}$ & 3.61 & 0.86 & 72.2 & High & 2 \\
\hline 6 & $\begin{array}{l}\text { Contributing to achieve } \\
\text { students' intellectual safety. }\end{array}$ & 3.28 & 0.97 & 65.6 & average & 8 \\
\hline 7 & $\begin{array}{l}\text { Using more than one } \\
\text { communication method to } \\
\text { express views and ideas. }\end{array}$ & 3.57 & 1.05 & 71.4 & High & 3 \\
\hline 8 & $\begin{array}{l}\text { Express opinions effectively } \\
\text { and fluently in front of all. }\end{array}$ & 3.04 & 1.17 & 60.8 & average & 9 \\
\hline 9 & $\begin{array}{l}\text { Providing safe and } \\
\text { support climate for } \\
\text { creative learning. }\end{array}$ & 3.29 & 1.13 & 65.8 & average & 7 \\
\hline General average & 3,43 & 0.96 & 68.6 & average & \\
\hline \hline
\end{tabular}

Table (7) results reveal that training needs degree for communication skill aspect is

average with mean of (3.43).

The greatest need was need (1)

"Organization of enrichment 
scientific material and present it in logical manner" with high degree and mean of (3.67). The lowest need was need (8) "Express opinions effectively and fluently in front of all" with average degree and mean of (3.04).

Fifth aspect: Cooperation, leadership and responsibility skill:

Table (8)

Study sample responses in Cooperation, leadership and responsibility skill $(\mathbf{n}=\mathbf{2 5})$

\begin{tabular}{l|l|c|c|c|c|c}
\hline \hline $\begin{array}{l}\text { S. } \\
\text { No }\end{array}$ & training needs & Mean & SD & $\begin{array}{l}\text { Relative } \\
\text { weight }\end{array}$ & $\begin{array}{l}\text { need } \\
\text { degree }\end{array}$ & Rank \\
\hline \hline 1 & $\begin{array}{l}\text { Knowing educational } \\
\text { standards for using } \\
\text { cooperative } \\
\text { strategies during teaching. }\end{array}$ & 4.46 & 1.23 & 89.2 & High & 4 \\
\hline 2 & $\begin{array}{l}\text { Carry out difficult tasks } \\
\text { that need to more creative } \\
\text { thinking in accomplishing } \\
\text { it }\end{array}$ & 4.49 & 1.11 & 89.8 & High & 3 \\
\hline 3 & $\begin{array}{l}\text { Exercise leadership team } \\
\text { through organization } \\
\text { of collective work. }\end{array}$ & 4.61 & 0.84 & 92.2 & High & 2 \\
\hline 4 & $\begin{array}{l}\text { Knowing profession } \\
\text { ethics of and work to } \\
\text { develop them in order to } \\
\text { achieve desired learning } \\
\text { outcomes. }\end{array}$ & 4.15 & 1.09 & 83.0 & High & 6 \\
\hline 5 & $\begin{array}{l}\text { Knowing leadership skills } \\
\text { through seminars } \\
\text { with specialists. }\end{array}$ & 4.72 & 0.97 & 94.4 & High & 1 \\
\hline 6 & $\begin{array}{l}\text { Knowing partnership } \\
\text { methods with } \\
\text { external community } \\
\text { organizations to develop } \\
\text { educational process. }\end{array}$ & 4.31 & 0.89 & 86.2 & High & 5 \\
\hline Overall average & 4.46 & 1.11 & 89.2 & High & \\
\hline \hline
\end{tabular}

Table (8) results reveal that training needs degree for Cooperation, leadership and responsibility skill aspect is high with mean of (4.46). The greatest need was need (5) "Knowing leadership skills through seminars with specialists" with high degree and mean of 
(4.72). The lowest need was need (4) "Knowing profession ethics of and work to develop them in order to achieve desired learning outcomes" with high degree and mean of (4.15).

Sixth aspect: Flexibility and Adaptability skill:

Table (9)

Study sample responses in flexibility and adaptability skill $(\mathrm{n}=\mathbf{2 5})$

\begin{tabular}{l|l|c|l|l|l|c}
\hline \hline $\begin{array}{l}\text { S. } \\
\text { No }\end{array}$ & training needs & Mean & SD & $\begin{array}{l}\text { Relative } \\
\text { weight }\end{array}$ & $\begin{array}{l}\text { need } \\
\text { degree }\end{array}$ & Rank \\
\hline \hline 1 & $\begin{array}{l}\text { Dealing with modern teaching } \\
\text { aids in teaching. }\end{array}$ & 3.67 & 1.11 & 73.4 & High & 5 \\
\hline 2 & $\begin{array}{l}\text { Knowing regulations and } \\
\text { laws that control educational } \\
\text { process. }\end{array}$ & 3.64 & 0.94 & 72.8 & High & 6 \\
\hline 3 & $\begin{array}{l}\text { Develop flexible plans on sound } \\
\text { scientific bases. }\end{array}$ & 4.17 & 1.15 & 83.4 & High & 1 \\
\hline 4 & $\begin{array}{l}\text { Modification of existing plans } \\
\text { based on hypothetical changes }\end{array}$ & 4.03 & 0.98 & 80.6 & High & 2 \\
\hline 5 & $\begin{array}{l}\text { Awareness of new curricula } \\
\text { importance and achievement } \\
\text { mechanisms. feedback to }\end{array}$ & 3.61 & 0.89 & 72.2 & High & 7 \\
\hline 6 & $\begin{array}{l}\text { Provide } \\
\text { modify shortcomings when } \\
\text { teaching students. }\end{array}$ & 3.73 & 1.15 & 74.6 & High & 4 \\
\hline 7 & $\begin{array}{l}\text { Provide immediate reinforcement } \\
\text { and support for correct learners' } \\
\text { responses. }\end{array}$ & 3.88 & 1.04 & 77.6 & High & 3 \\
\hline General average & 3.82 & 0.98 & 76.4 & High & \\
\hline
\end{tabular}

Table (9) results reveal that training needs degree for flexibility and adaptability skill aspect is high with mean of (3.82). The greatest need was need (3) "Develop flexible plans on sound scientific bases" with high degree and mean of (4.17). The lowest need was need (5) "Awareness of new curricula importance and achievement mechanisms" with high degree and mean of (3.61).

Seventh aspect: selfdirecting and productivity sk ill: 
Table (9)

Study sample responses in self- directing and productivity skill $(\mathbf{n}=\mathbf{2 5})$

\begin{tabular}{l|l|c|l|c|c|c}
\hline \hline $\begin{array}{l}\text { S. } \\
\text { No }\end{array}$ & training needs & Mean & SD & $\begin{array}{l}\text { Relative } \\
\text { weight }\end{array}$ & $\begin{array}{l}\text { need } \\
\text { degree }\end{array}$ & Rank \\
\hline \hline 1 & $\begin{array}{l}\text { Identify strengths and weaknesses through } \\
\text { the analysis of self-evaluation tools. }\end{array}$ & 4.56 & 1.12 & 91.2 & High & 3 \\
\hline 2 & $\begin{array}{l}\text { Using available environmental } \\
\text { resources in implementation of } \\
\text { students' activities. }\end{array}$ & 4.53 & 0.89 & 90.6 & High & 4 \\
\hline 3 & $\begin{array}{l}\text { Knowing student projects that contribute in } \\
\text { achieving curricula goals. }\end{array}$ & 4.35 & 0.96 & 87.0 & High & 6 \\
\hline 4 & $\begin{array}{l}\text { Knowing objective ways to use self- } \\
\text { evaluating tools to achieve its goals. }\end{array}$ & 4.48 & 0.91 & 89.6 & High & 5 \\
\hline 5 & $\begin{array}{l}\text { Organizing productive projects that suit } \\
\text { students' interests. }\end{array}$ & 4.63 & 1.15 & 92.6 & High & 2 \\
\hline 6 & $\begin{array}{l}\text { Knowing analysis mechanism of education } \\
\text { reality forms and benefit from it }\end{array}$ & 4.75 & 1.04 & 95.0 & High & 1 \\
\hline Overall average & 4.49 & 1.09 & 89.8 & High & \\
\hline \hline
\end{tabular}

\section{Table (10) results} reveal that training needs degree for self-directing and productivity skill aspect is high with mean of (4.49). The greatest need was need (6) "Knowing analysis mechanism of education reality forms and benefit from it" with high degree and mean of (4.75). The lowest need was need (3) "Knowing student projects that contribute in achieving curricula goals." with high degree and mean of (4.35).

To identify university staff members' training needs in light of 21 st century skills y in general, researcher calculated means and standard deviations and relative weight for questionnaire aspects as a whole as seen by study sample, the following table illustrates this:

\section{Table (11)}

Study sample responses for the questionnaire aspects as a whole $=\mathbf{2}$

\begin{tabular}{l|l|c|c|c|c|c}
\hline \hline $\begin{array}{l}\text { S. } \\
\text { No }\end{array}$ & training needs & Mean & SD & $\begin{array}{l}\text { Relative } \\
\text { weight }\end{array}$ & $\begin{array}{l}\text { need } \\
\text { degree }\end{array}$ & Rank \\
\hline \hline 1 & Innovation and creativity skill. & 4.17 & 1.04 & 83.4 & Hig & 5 \\
\hline 2 & $\begin{array}{l}\text { Critical thinking } \\
\text { and problem solving skill. }\end{array}$ & 4.29 & 1.01 & 85.8 & High & 4 \\
\hline 3 & $\begin{array}{l}\text { Information and } \\
\text { technological culture skill. }\end{array}$ & 4.67 & 0.89 & 93.4 & High & 1 \\
\hline \hline
\end{tabular}


Follow Table (11)

Study sample responses for the questionnaire aspects as a whole $=\mathbf{2}$

\begin{tabular}{l|l|c|l|l|c|c}
\hline \hline $\begin{array}{l}\text { S. } \\
\text { No }\end{array}$ & training needs & Mean & SD & $\begin{array}{l}\text { Relative } \\
\text { weight }\end{array}$ & $\begin{array}{l}\text { need } \\
\text { degree }\end{array}$ & Rank \\
\hline \hline 4 & Communication skill. & 3.43 & 0.96 & 68.6 & Average & 7 \\
\hline 5 & $\begin{array}{l}\text { Cooperation, leadership and } \\
\text { responsibility skill. }\end{array}$ & 4.46 & 1.11 & 89.2 & High & 3 \\
\hline 6 & $\begin{array}{l}\text { Flexibility and } \\
\text { adaptability skill. }\end{array}$ & 3.82 & 0.98 & 76.4 & High & 6 \\
\hline 7 & $\begin{array}{l}\text { Self- directing } \\
\text { and productivity skill. }\end{array}$ & 4.49 & 1.09 & 89.8 & High & 2 \\
\hline \multicolumn{2}{l|}{ General average } & 4,19 & 0.99 & 83.8 & High & \\
\hline \hline
\end{tabular}

Table (11) results Ishaq, 2011 \& Abdullah \& Abo reveal that staff members training needs in light of $21 \mathrm{st}$ century skills degree in general is high, with general relative weight of $(83.8 \%)$ and aspect rates ranged between $(68.6 \%$ and $93.4 \%$ ) This confirm the necessity for determine university staff members training needs in light of $21 \mathrm{st}$ century skills and train on it

Researcher attribute that "information and technological culture skills" aspect is the greatest staff member need to the current era nature, which require every staff member to know and master computer skills and different information sources in a way can support and enhance his professional performance. This result is in line with (Al-Buhairi, 2011;

Elfadl, 2014) results that confirmed priority and importance for training staff members on technological skills, which suit current era. In addition to give training to university's staff members on how to use technology in higher education to enable them to perform their roles in a way can help in improve educational process in universities.

As well as giving attention to life and social skills such as adaptation, flexibility, leadership, cooperation and productivity, which are of university staff member's success factors.

Critical and creative thinking, which is a main and base 
objective of all curricula, and every staff member, should know how to develop it within students while teaching his specialization, the matter, which confirm necessity to pay attention to develop this skills within staff members firstly and train them how to develop this skills within students.

\section{For communication} skill, which was the lowest need, even its relative weight was high $(68 \%)$. This may be due that communication skill with all its sub-skills like reading and writing skills, speaking and listening skills available in an acceptable degree for most staff members, and other training needs are more required

\section{II- second query results:}

To answer this query and testing first hypothesis independent " $T$ " test used to find out differences between staff members responses to training need in light of $21 \mathrm{st}$ century skills according to qualification variable, as illustrated in the table below :

\section{Table (12)}

\section{Means and standard deviations and " $T$ " value for differences in} staff members responses according to qualification variable PhD (n=14), Master (n=11)

\begin{tabular}{l|l|l|l|l|l|l|l}
\hline \hline \multirow{2}{*}{$\begin{array}{l}\text { S. } \\
\text { No }\end{array}$} & Aspect & \multicolumn{2}{l|}{ PhD } & \multicolumn{2}{|l|}{ Master } & \multicolumn{2}{|l}{$\begin{array}{l}\text { Significance } \\
\text { value }\end{array}$} \\
\cline { 3 - 8 } & Mean & SD & Mean & SD & \\
\hline \hline 1 & Innovation and creativity skill. & 4.13 & 0.97 & 4.21 & 1.04 & 0.189 & Insignificant \\
\hline 2 & $\begin{array}{l}\text { Critical thinking } \\
\text { and problem solving skill. }\end{array}$ & 4.24 & 1.09 & 4.34 & 0.92 & 0.239 & Insignificant \\
\hline 3 & $\begin{array}{l}\text { Information and } \\
\text { technological culture skill. }\end{array}$ & 4.58 & 1.01 & 4.76 & 0.89 & 0.454 & Insignificant \\
\hline 4 & Communication skill. & 3.37 & 0.95 & 3.49 & 1.11 & 0.274 & Insignificant \\
\hline 5 & $\begin{array}{l}\text { Cooperation, leadership and } \\
\text { responsibility skill. }\end{array}$ & 4.44 & 0.88 & 4.48 & 0.94 & 0.104 & Insignificant \\
\hline 6 & $\begin{array}{l}\text { Flexibility and } \\
\text { adaptability skill. }\end{array}$ & 3.77 & 1.07 & 3.87 & 1.02 & 0.228 & Insignificant \\
\hline 7 & $\begin{array}{l}\text { Self- directing } \\
\text { and productivity skill. }\end{array}$ & 4.46 & 0.97 & 4.52 & 0.89 & 0.154 & Insignificant \\
\hline General average & 4.14 & 1.04 & 4.24 & 0.97 & 0.238 & Insignificant \\
\hline \hline
\end{tabular}

(T) significant at (0.05) level and $24 \mathrm{DF}=2.07$
Table
(12) results
difference
according
to qualification at 0.05 level in all

reveal statistically insignificant 
study aspects. This result is in line with (Al-Buhairi, 2011; Hamdan, 2017) results where there was no statistically significant differences in training needs between staff members according to qualification. In same time this result differs with (Ishaq , 2011; Abdullah \& Abo Elfadl, 2014) results where there were statistically significant differences in training needs between staff members according to qualification towards Master degree staff.

Although there were no significant differences in training needs for staff members in all aspects according to qualification, Master degree holders were in more need for training in light of 21 st century skills in each aspect and in overall degree.

Table (13)

Means and standard deviations and " $T$ " value for differences in staff members responses according to scientific department variable Granting departments $(n=16)$, non-granting departments $(n=91)$

\begin{tabular}{c|l|c|c|c|c|c|c}
\hline \hline \multirow{2}{*}{$\begin{array}{c}\text { S. } \\
\text { No }\end{array}$} & \multicolumn{1}{|c|}{ Aspect } & \multicolumn{2}{|c|}{$\begin{array}{c}\text { Granting } \\
\text { Departments }\end{array}$} & \multicolumn{2}{|c|}{$\begin{array}{c}\text { Non- } \\
\text { granting } \\
\text { Departments }\end{array}$} & $\begin{array}{c}\text { T } \\
\text { value }\end{array}$ & $\begin{array}{c}\text { Significance } \\
\text { Level }\end{array}$ \\
\cline { 3 - 7 } & & Mean & SD & Mean & SD & & \\
\hline \hline 1 & Innovation and creativity skill. & 4.24 & .97 & 4.10 & 0.88 & 0.343 & Insignificant \\
\hline 2 & $\begin{array}{l}\text { Critical thinking } \\
\text { and problem solving skill. }\end{array}$ & 4.37 & .04 & 4.21 & 0.96 & 0.364 & Insignificant \\
\hline \hline
\end{tabular}

Researcher attribute this to that $\mathrm{PhD}$ holders acquire experience and modern methods, which help in facilitating their teaching. In addition to knowing about recent cognition trends and their ability to benefit from and apply it in different fields in the form, which helps them to develop their teaching practices, which is not available for Master degrees holders, and so First hypothesis accepted.

To test second hypothesis independent " $\mathrm{T}$ " test used to find out differences between staff members responses to training need in light of 21st century skills according to scientific department variable, as illustrated in the table below: 


\section{2}

Follow Table (13)

Means and standard deviations and " $T$ " value for differences in staff members responses according to scientific department variable Granting departments $(n=16)$, non-granting departments $(\mathbf{n = 9 1})$

\begin{tabular}{c|l|c|c|c|c|c|c}
\hline \multirow{2}{*}{$\begin{array}{c}\text { S. } \\
\text { No }\end{array}$} & \multicolumn{1}{|c|}{ Aspect } & \multicolumn{2}{|c|}{$\begin{array}{c}\text { Granting } \\
\text { Departments }\end{array}$} & \multicolumn{2}{|c|}{$\begin{array}{c}\text { Non- } \\
\text { granting } \\
\text { Departments }\end{array}$} & $\begin{array}{c}\text { T } \\
\text { value }\end{array}$ & $\begin{array}{c}\text { Significance } \\
\text { Level }\end{array}$ \\
\cline { 3 - 7 } & & Mean & SD & Mean & SD & & \\
\hline \hline 3 & $\begin{array}{l}\text { Information and } \\
\text { technological culture skill. }\end{array}$ & 4.72 & .87 & 4.62 & 0.91 & 0.260 & Insignificant \\
\hline 4 & Communication skill. & 3.53 & .92 & 3.31 & 1.06 & 0.521 & Insignificant \\
\hline 5 & $\begin{array}{l}\text { Cooperation, } \\
\text { leadership and } \\
\text { responsibility skill. }\end{array}$ & 4.48 & 0.99 & 4.44 & 0.92 & 0.091 & Insignificant \\
\hline 6 & $\begin{array}{l}\text { Flexibility and } \\
\text { adaptability skill. }\end{array}$ & 3.95 & 1.08 & 3.69 & 0.94 & 0.580 & Insignificant \\
\hline 7 & $\begin{array}{l}\text { Self- directing } \\
\text { and productivity skill. }\end{array}$ & 4.51 & 0.97 & 4.47 & 1.03 & 0.093 & Insignificant \\
\hline General average & 4.26 & 0.93 & 4.12 & 1.01 & 0.333 & Insignificant \\
\hline \hline
\end{tabular}

(T) significant at (0.05) level and $24 \mathrm{DF}=2.07$

Table (13) results between staff members reveal statistically insignificant difference according to scintefic department (granting, non-granting) at 0.05 level in all study aspects. This result is in line with (Ishaq , 2011; AlBuhairi, 2011; Abdullah \& Abo Elfadl, 2014) results where there was no statistically significant differences in training needs between staff members according to sintific department. In same time this result differs with (Al-Sharif, 2006) results where there were statistically significant differences in training needs according to scientific department.

Although there were no significant differences in training needs for staff members in all aspects according to scientific department, Staff members in granting departments were in more need for training in light of 21 st century skills in each aspect and in overall degree.

Researcher attributes this to training needs importance in granting departments because of the educational experience and life skills they give to their

Assiut Journal For Sport Science Arts 
students in order to prepare and qualify them to engage in labor market, deal with and upgrading society. Thus to carry out tasks assigned to them, whether through teach ing for future generations, preparing distinct players pain and qualify them to participate in higher level local and international tournaments. Also through dealing with sport institutions in society, aiming to upgrade it to be able to deal and face $21 \mathrm{st}$ century requirements. So second hypothesis accepted.

\section{Recommendations and proposals:}

In light of study results, the following could be recommended:

1- Considering training needs reached in current study as objectives of any training program that university seeks to implement it for developing staff members performance in light of 21 st century skills

2- Design training programs for staff members at Faculty of Physical Education, Al-Azhar University in light of training needs in this study in light of 21 st century skills according to their priority and importance in staff members opinion

\section{3- Preparing}

comprehensive training plan to develop staff members skills based on accurate identification of training needs accordance to scientific methods, taking into account future changes to build training programs in light of new needs and future roles of staff member.

4- Activating distance-

training methods for staff members, using modern technology in a way ensuring interaction, goals achievement, and improving economic feasibility for presented training programs.

5- Conducting an evaluative study to measure the effect of training courses for staff members based on their needs on their performance.

\section{References}

1- Abdulkader, M. (2014). Redirecting teachers professional development in light of $21^{\text {st }}$ century skills. Faculty of education journal, Al-Azhar University, 156 (4), 671-794.

2- Abdullah, H. \& Abo Elfadl, A. (2014). Training needs of staff members at 
Aljouf University from their point of view in light of professional requirements and some variables. Faculty of Education journal, Al-Azhar University, 161(1), 896-935

3- Al-Buhairi, M. (2011). Training needs of university staff members in Elearning field as seen by staff members at Faculty of Education, King Khalid University. Reading and Knowledge Magazine, Faculty of Education, Ain Shams University, (115), 134-160

4- Al-Dahshan, G. \& Badran, S. (2001) Renewal in Higher Education. Cairo: Dar Qabaa for Printing and Publishing.

5- Alnabawy, A. \& Galal. A. (2016). The role of higher education institutions in Oman to provide its graduates the skills and knowledge of the twenty-first century. Global Institute for Study \& Research Journal (GISR-J), 2(2), 18-34.

6- Al-Sharif, M. (2006). Training needs for staff members at Taif University, Journal of Studies in Curricula and Teaching Methods, Faculty of Education, Ain Shams University, (117), 190-224.

7- Badr, S. (2015). Training requirements for university staff members in light of effective teaching skills in Faculty of Science, Princess Nora bent Abdulrahman University.

International Interdisciplinary Journal of Education, Jordan 4(6), 183-202.

8- Bashir, M. (2004). Professional development of university staff members: comparative study. Cairo: Books world.

9- Chuang, W. (2002). An innovative teacher training approach: combine live instruction with a web - based reflection system. British Journal of Educational Technology, 33 (2), 229-232. doi: $\quad 10.1111 / 1467-8535$. 00256.

10- Hamdan, A. (2017) Palestine universities staff members training needs: Ismaic university-case study. Journal of Islamic University for Educational and Psychological Studies, Islamic University- Gaza, 25(2), 347369

11- Ishaq, A. (2011). Training needs of staff members at University College in Makkah in field of Educational Technology Innovations, Journal of Studies in 
Curriculum and Educational Supervision, Saudi Scientific Society for curricula and educational supervision, Umm Al-Qura university. 3(1), 1757.

\section{2- Kayange, J. J., $\quad$ \&} Msiska, M. (n.d.). Teacher education in china: training teachers for the 21st century. The online journal of new horizons in education, 6(4), 204-210. Retrieved from https://www. tojned.net/journals/tojned/articl es/v06i04/v06i0424.pdf.

13- Mahmoud, M. (2001). 21 st century teacher - choosing and preparation - in light of Islamic orientations. Cairo: Arab Thought House.

14- Mahmoud, Y. (2009). A new vision for higher education development. Cairo: Egyptian Lebanese house.

15- Mishra, S. (2007). Quality assurance in higher education: An introduction. Dhaka, India: National printing press.
16- Morrison, G. R., \& Lowther, D. L. (2002). Integrating computer technology into the classroom. Upper Saddle River, NJ: Prentice Hall.

17- Sadiq, M. (2014) Training planning and its role in achieving public and private organizations aims. Cairo: Arab Group for training and Publishing.

18- Sahu, R. K. (2010). Group dynamics and team building. New Delhi, India: Excel books. 19- Shalabi, N. (2014). Proposed framework integrate $21^{\text {st }}$ century skills in science curricula in basic learning stage in Egypt. International Interdisciplinary Journal of Education, Jordan 3(10), 1-33.

20- Teima, R. \& Albandary, M. (2004). Higher Education: Between monitoring reality and development visions. Cairo: Arab thought house..

21- Trilling, B., \& Fadel, C. (2009). 21st century skills: Learning for life in our times. Hoboken, NJ: John Wiley \& Sons.. 